\title{
ОБРАБОТКА ИЗОБРАЖЕНИЙ С ПОМОЩЬЮ НЕЧЕТКОЙ СЕГМЕНТАЦИИ В МЕДИЦИНСКОЙ ДИАГНОСТИКЕ
}

\author{
Ф.Н. Бузылев, С. Н. Щербакова, Е. А. Бородин \\ ФЕДОР НИКОЛАЕВИЧ БУЗЫЛЕВ - к.т.н., дочент РТУ МИРЭА. E-mail: buzylev@mirea.ru. \\ СВЕТЛАНА НИКОЛАЕВНА ЩЕРБАКОВА - старший преподаватель РТУ МИРЭА. \\ E-mail: Shcherbakova@mirea.ru. \\ ЕВГЕНИЙ АЛЕКСАНДРОВИЧ БОРОДИН - бакалавр РТУ МИРЭА. E-mail: borodin.gene@gmail.com. \\ 119454, г. Москва, Проспект Вернадского, д. 78, Федеральноегосударственное бюджетное образовательное \\ учреждение высшего образования «МИРЭА - Российский технологический университет» (РТУ МИРЭА).
}

В статье рассмотрены проблемы, возникающие во время сегментирования при обработке изображений. Приводится общая стратегия при проведении кластеризации как основного метода сегментации. Показан подход к обработке медииинских изображений с помощью нечеткой кластеризаиии на основе алгоритма FCM, проведено сравнение алгоритма FCM с более традиционным алгоритмом четкой кластеризации K-средних, с видимьлми преимуществами алгоритма нечеткой кластеризации.

Ключевые слова: обработка изображений, нечеткая кластеризация, метод К-средних.

\section{FUZZY SEGMENTATION BASED DIGITAL PROCESSING IN MEDICAL DIAGNOSTICS}

\author{
F. N. Buzylev, S. N. Shcherbakova, E.A. Borodin \\ 78 Vernadsky Avenue, Moscow 119454. MIREA - Russian Technological University (RTU - MIREA).
}

The article defines what image segmentation is, the role of segmentation in applications, examples of the use of segmentation. Problems with image processing using segmentation are noted. The general strategy for clustering as the main method of segmentation is outlined. An approach to the processing of medical images using fuzzy clustering based on the FCM algorithm is shown, the FCM algorithm is compared with the more traditional $K$-means crisp clustering algorithm, with the visible advantages of the fuzzy clustering algorithm.

Keywords: digital processing, fuzzy clustering, K-means algorithm. 
Сегментация изображения - это процесс разделения изображения на неперекрывающиеся области, которые являются однородными по некоторым характеристикам, таким как интенсивность, цвет или текстура. Сегментация изображений это фундаментальный вопрос для систем компьютерного зрения и понимания изображений, необходимый во многих приложениях, таких как распознавание и отслеживание объектов/образов, поиск изображений и так далее. Другими словами, цель сегментации состоит в том, чтобы упростить и/или изменить представление изображения в нечто более значимое и более простое для анализа. Сегментация изображения обычно используется для определения местоположения объектов и границ (линий, кривых и т. д.) на изображениях. Точнее говоря, сегментация изображения - это процесс назначения метки каждому пикселю изображения, чтобы пиксели с одинаковой меткой имели определенные характеристики.

Проблема сегментации изображений все еще далека от удовлетворительного решения. Производительность каждого метода сильно зависит от типа визуальных сцен и от параметров изображения, таких как разрешение, освещенность и визуальные условия. Фундаментальные методы сегментации обнаруживают различия между пикселями разных областей или сходство среди пикселей одной и той же области. Один из основных методов сегментации - кластеризация.

Кластеризация - это процесс группировки набора пикселей - в подмножества (называемые кластерами), так что точки в одном кластере в некотором смысле похожи. Общей стратегией является разделение изображения на несколько блоков и извлечение ряда локальных признаков для каждого блока. Затем к этим признакам применяется алгоритм кластеризации, и полу- чается заранее определенное количество кластеров. Для сегментации изображения были предложены как четкие, так и нечеткие схемы кластеризации. Нечеткие методы показали более надежные результаты, чем четкие алгоритмы, особенно в случае изображений, характеризующихся некоторой неоднозначностью, такой как низкая контрастность, шум и негомогенность. Среди алгоритмов нечеткой кластеризации наиболее распространенным является известный алгоритм Fuzzy C-Means (FCM). Несмотря на широкое использование, FCM не всегда обеспечивает хорошие результаты сегментации из-за того, что он не включает в себя какую-либо информацию, касающуюся пространственного контекста, поскольку полученные области могут быть непересекающимися, нерегулярными, шумными и т.д.

В качестве примера реализуем четкую (стандартный алгоритм K-means) и нечеткую (FCM) сегментацию изображений МРТ, полученных от пациентов с опухолями головного мозга.

Прежде чем мы перейдем к какой-либо кластеризации, мы просмотрим наше изображение на рис. 1.

Для того чтобы кластеризация была эффективной, необходимо четко различать «группировки» интенсивности пикселей. Построим гистограмму интенсивности пикселей в зависимости от количества пикселей (ось х), имеющих эту интенсивность (ось у), и посмотрим, как это выглядит на рис. 2.

Мы видим несколько пиков в нашей гистограмме, поэтому кластеризация может дать хороший результат. В распределении интенсивности пикселей может быть 3-4 кластера, значит попробовать столько кластеров в алгоритме кластеризации.

Стандартный алгоритм Kmeans (пакет sci-kit learn Kmeans, количество кластеров (равное 3)).
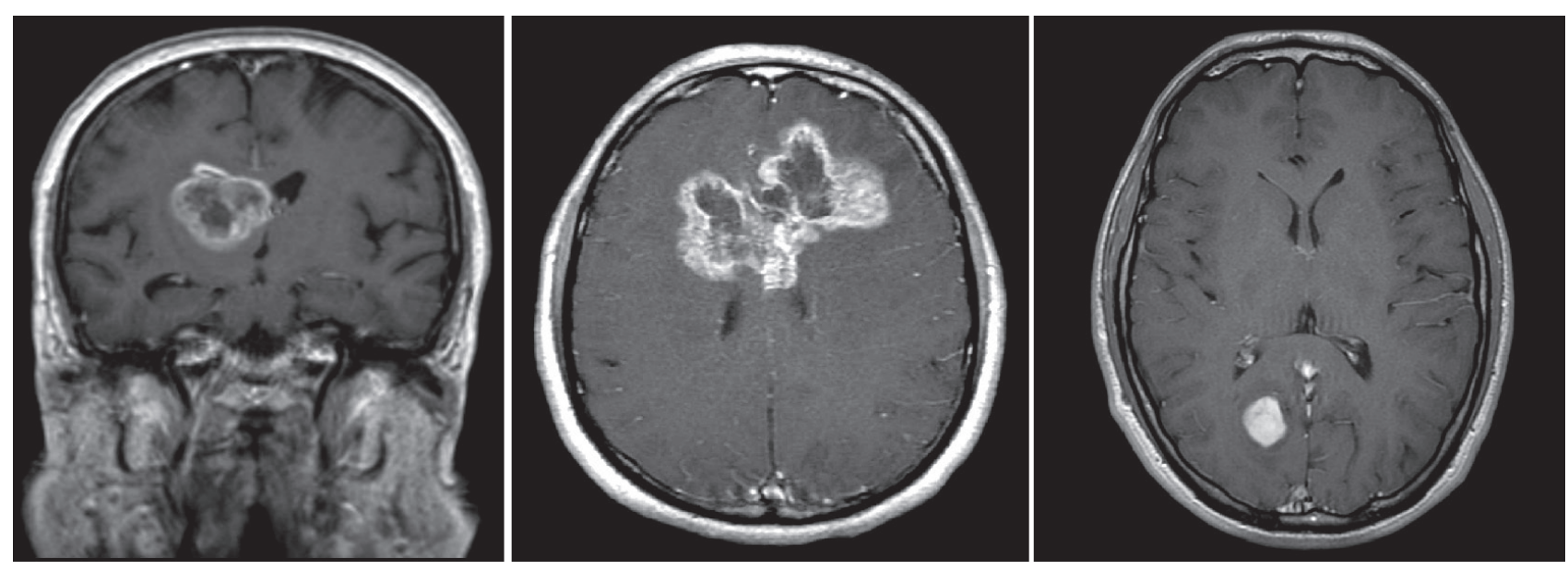

Рис. 1. Изображения МРТ опухолей 

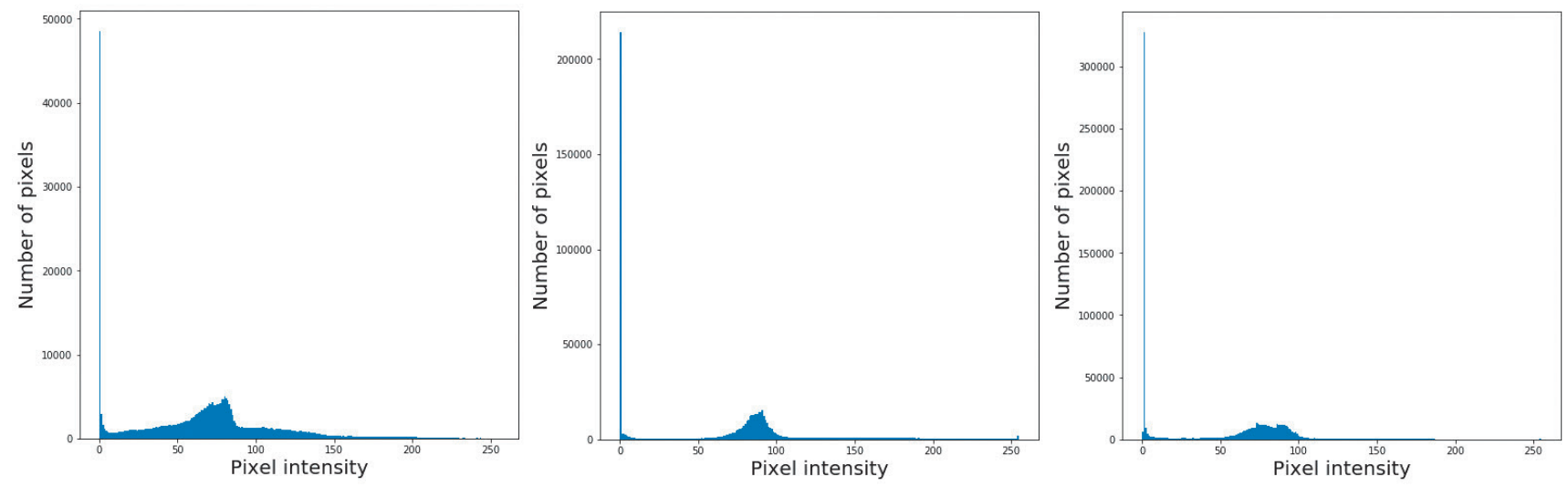

Рис. 2. Гистограмма уровней серого
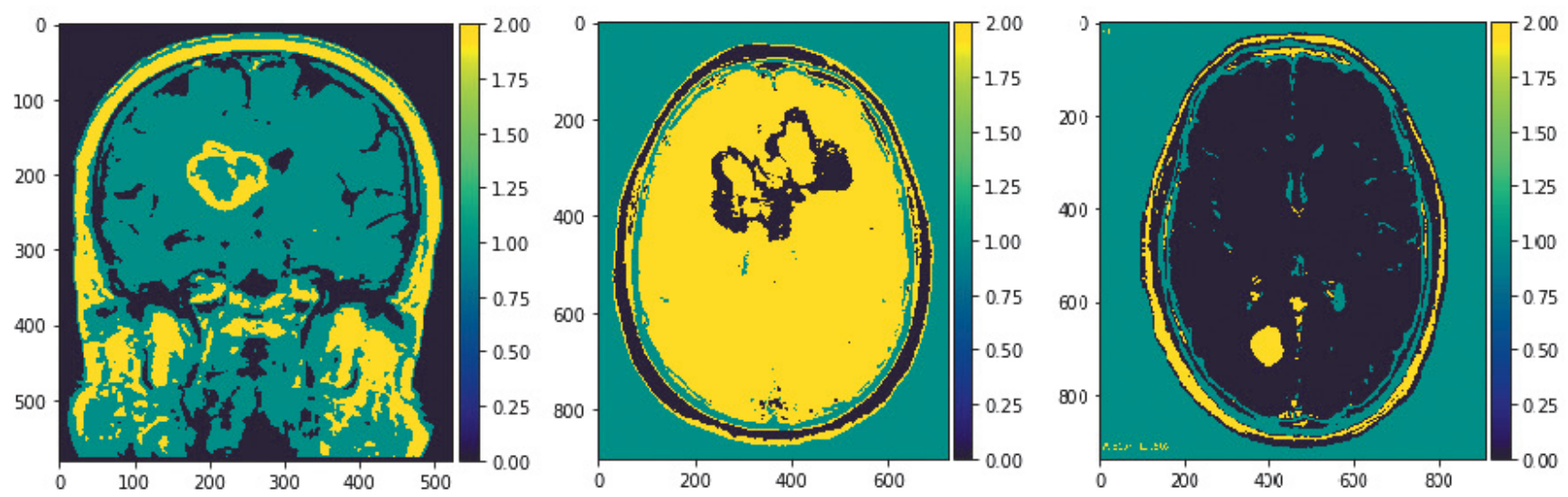

Рис. 3. Результат работы метода четкой кластеризации
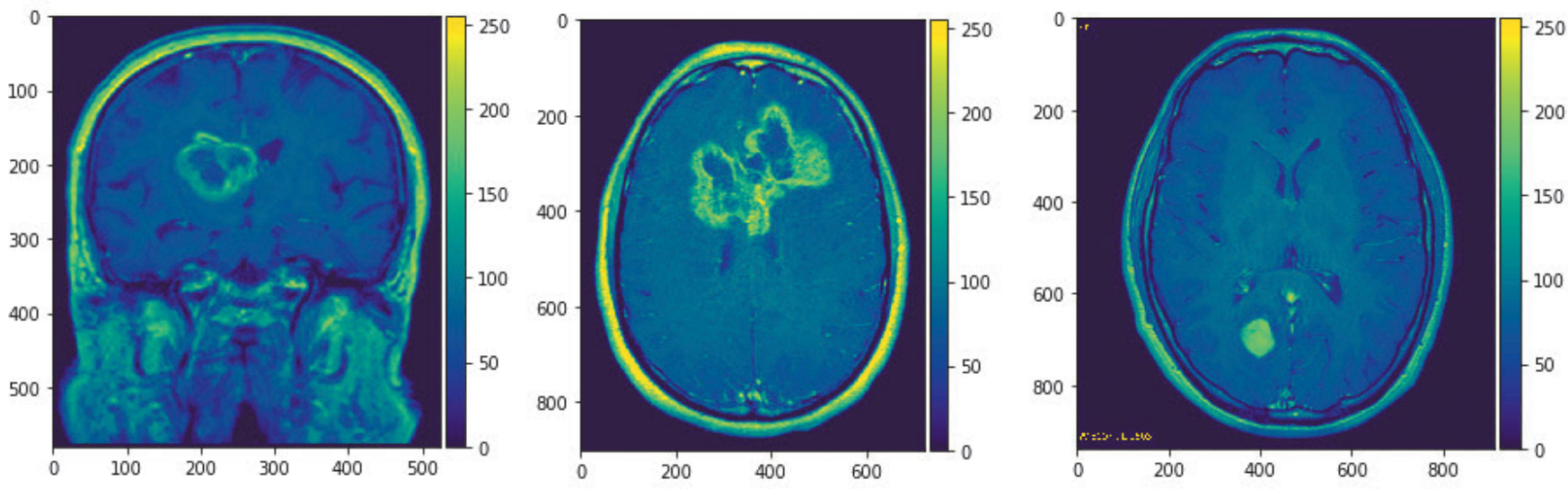

Рис. 4. Результат работы метода нечеткой кластеризации

Мы видим три цвета (темно-синий, бирюзовый и желтый), каждый из которых соответствует трем интенсивностям, которые мы определили в нашем анализе выше. Мы четко видим очертания опухоли в центре изображения.
После стандартной кластеризации Kmeans, посмотрим на результат нечеткой кластеризации.

Для реализации нечеткой кластеризации мы будем использовать пакет sci-kit, называемый skfuzzy. (https://pythonhosted.org/scikit-fuzzy/). 
Результат работы этого метода FCM приведен на рис. 4.

Видна большая ясность полученного результата, также не потеряны данные, которые мы могли бы снова использовать в качестве входных для дополнительной обработки изображений с использованием пороговых методов, обнаружения краев с помощью LoG и т. д.

\section{Лuтература}

1. «Medical image processing», T.Chaira, CRC Press, 2015.

2. «Fuzzy Logic for Image Processing. A Gentle Introduction Using Java», L. Caponetti and G. Castellano; Springer, 2017.

3. «Fuzzy sets for image processing and understanding», I. Bloch, Institut Mines-Telecom, Telecom ParisTech, CNRS LTCI, Paris, France, 2015. 